\title{
ÉTICA Y HUMANISMO EN LA FORMACIÓN MÉDICA
}

\begin{abstract}
Alberto Perales Cabrera*
Resumen: El reclamo de enfatizar los ejes ético y humanista en la formación médica del siglo XXI se apoya no sólo en la observación repetida de conductas profesionales inadecuadas de los médicos en ejercicio, sino también de inequidades en el sistema de atención de salud que, frecuentemente, violan los derechos de los pacientes y generan reclamos de la sociedad. Un esfuerzo serio para mejorar la calidad ética y humanista de la formación médica debe insertarse en una adecuada teoría del desarrollo moral del hombre y partir de una reflexión crítica sobre los postulados que sustentan tal formación, los profesores que la imparten y los procedimientos y escenarios que se utilizan para ese fin.
\end{abstract}

Palabras clave: formación médica, humanismo, desarrollo moral

\section{ETHICS AND HUMANISM IN MEDICAL'S TRAINING}

\begin{abstract}
The claim to emphasize the ethic and humanistic axes of the medical education in the XXIst century leans not only on the repeated observation of doctors' professional misconducts, but also on the System of Health Care's inequities which often violate the patients' rights and arise society's complaints. A serious effort must be done to improve the ethic and humanistic medical training which must be inserted in an adequate theory of man's moral development, and start from a critical reflection about the requests that underlie that training, the professors and the procedures and sceneries that are employed to reach that aim.
\end{abstract}

Key words: medical training, humanism, moral development

\section{ÉTICA E HUMANISMO NA FORMAÇÃO MÉDICA}

Resumo: A exigência de enfatizar o eixo ético e humanista na formação médica do século XXI, se apóia não somente na observação repetida de condutas profissionais inadequadas dos médicos em exercício mas, também, de iniqüidades no sistema de atenção de saúde que, frequentemente, violam os direitos dos pacientes e geram protestos da sociedade. Um esforço sério para melhorar a qualidade ética e humanista da formação médica deve se inserir numa adequada teoria de desenvolvimento moral do homem, bem como, os professores que a partilham e os procedimentos que se utilizam para este fim, aprofundar uma uma reflexão crítica sobre os postulados que sustentam tal formação.

Palavras chave: formação médica, humanismo, desenvolvimento moral

* Director del Instituto de Ética en Salud y del Comité de Ética de Investigación de la Facultad de Medicina, Universidad Nacional Mayor de San Marcos, Perú

Correspondencia: perales.alberto@speedy.com.pe 


\section{Introducción}

En educación médica se aspira al logro de tres fines: a) cognitivos, mediante la transmisión de conocimientos, para enseñar al alumno cómo, cuándo y por qué realizar el acto y los procedimientos médicos; b) de destrezas y habilidades, para adiestrarlo en la forma adecuada de realizarlos, y c) de actitudes y conducta profesional, para que desarrolle el comportamiento que corresponde al trabajo médico(1).

Para el primero de estos fines, la buena información científica y humanística general es indispensable. Actualmente, con la revolución informacional, tal insumo puede obtenerse con menor presencia del profesor, quien, en consecuencia, ha variado su rol de fuente de conocimiento experto a modulador sensato del sedimento que el estudiante hará de esa información. Por ello, actualmente se suele enfatizar en la capacitación tecnológica del alumno para que capte tal insumo en la forma más completa posible.

En relación con el segundo objetivo, la presencia del profesor es aún necesaria. La transferencia de destrezas y habilidades por medio de prácticas supervisadas permite al estudiante adquirir los procedimientos clínicos indispensables para el diagnóstico y tratamiento de diversas enfermedades.

Finalmente, cuando de actitudes y conductas profesionales se trata, se requiere no sólo de la presencia del profesor sino de tiempo, espacio y condiciones necesarias para el establecimiento de una relación emocional específica entre docente y discente. Sin esta esencial condición, la posibilidad de que aquél influya en el modelaje de la conducta profesional del estudiante se torna incierta.

La educación médica que se imparte en las universidades peruanas cumple, con dificultad, los dos primeros objetivos y fracasa claramente en el tercero, salvo excepciones.

El reclamo mundial de enfatizar los ejes ético (como ciencia de la moral) y humanista (referido al conjunto de ideas que expresan respeto hacia la dignidad humana y preocupación por el bien de los hombres) en la formación del médico del siglo XXI se apoya no sólo en la observación repetida de conductas profesionales inadecuadas de los médicos en ejercicio, sino también de inequidades en el sistema de atención de salud que, frecuentemente, violan los derechos de los pacientes y generan reclamos de la sociedad.

\begin{tabular}{l}
\hline VIOLACIÓN DE LOS DERECHOS DEL PACIENTE \\
\hline Se le brinda información médica incompleta. \\
No se aplica o se aplica deficientemente el consentimiento \\
informado. \\
No se respeta su privacidad. \\
No siempre se protege la confidencialidad de sus datos. \\
La consulta hospitalaria es de insuficiente duración y de menor \\
calidad. \\
Se ofrece un inadecuado sistema de citas con largas filas de espera. \\
Maltrato, con atención desconsiderada e irrespetuosa.
\end{tabular}

Los griegos demandaban a las profesiones básicas, tales como la política (de acuerdo con Platón, la ciencia del gobierno de los pueblos), el sacerdocio, la medicina y las que se ocupaban de la Ley, basarse en la ética. Entre todas éstas, la medicina siempre destacó por tal preocupación. Pero lo ético no se da en el vacío. Lo que calificamos como tal es un tipo de conducta humana que se ajusta a las normas sociales establecidas para la convivencia constructiva y pacífica, con el fin de lograr, como afirmaba Aristóteles, el bien supremo de la felicidad.

En ese sentido, la conducta ética no depende exclusivamente de un actor (en este caso, del estudiante de medicina) sino que, como toda conducta humana, está

\section{RECLAMOS SOCIALES A LA PROFESIÓN MÉDICA}

Descontento general, con merma de su prestigio social.

Acusaciones de deshumanización y mercantilismo al sistema de atención de salud.

Injerencia de terceras partes en el sistema de salud que han alterado el acto médico.

Hospitales con equipos insuficientes y desactualizados.

Costos altos.

Menor mística en las instituciones formadoras con visibles intereses mercantilistas.

determinada por múltiples factores situacionales del entorno en que se desarrolla.

Nuestra tesis fundamental es que la formación ética y humanista del discente dependerá del impacto que sobre él/ella produzca el sistema formador, es decir, la ética institucional donde se ha formado (o deformado) a lo largo de siete o más ańos de su existencia, parte de la cual transcurre durante su ciclo vital de adolescente.

\section{La formación ética y humanista de los alumnos de medicina}

Existe consenso entre los educadores médicos respecto de la necesidad de formación ética y humanista del estudiante de medicina. El problema es ¿cómo? 


\section{¿Qué entendemos por sistema formador?}

Un sistema es definido como el conjunto de elementos interconectados por relaciones de función y dependencia, por lo cual, cada nivel de integración o subsistema es influenciado por los otros niveles. El enfoque sistémico permite comprender que en toda conducta o reacción humana (sea en situación de salud o de enfermedad) la causalidad no es lineal ni obedece a un único factor sino que, por el contrario, es determinada por una variedad de factores provenientes de los diversos niveles del sistema en los cuales el sujeto existe.

En nuestro concepto de "sistema formador", incluimos como niveles a la sociedad, la universidad, la facultad de medicina, las sedes docentes hospitalarias, el cuerpo docente, el cuerpo administrativo y los alumnos. Todos ellos ejercen diversos grados de influencia en el discente como ser humano individual.

En esta óptica, distinguimos dos formas de entender la formación ética del estudiante de medicina:

- Como enseñanza curricular, impartida como información teórica por medio de cursos específicos sobre Filosofía de la Medicina, Ética, Deontología y Bioética, y a través de seminarios, talleres, foros, discusión de casos clínicos con problemas éticos y otros.

- Como desarrollo moral de la personalidad. Destacamos la importancia básica de la relación emocional docente-discente y el valor del "buen ejemplo" que produce aprendizaje vicario en el estudiante. Todo ello en el marco apropiado de una ética institucional consistente y madurativa, en la cual haya, además, cumplimiento transparente de las normas institucionales por parte de las autoridades, los profesores, personal en general y, por supuesto, los alumnos.

Nuestra impresión es que, en general, los sistemas formadores han considerado erróneamente que la formación humanista y ética del estudiante depende sólo de la primera forma citada. Por ejemplo, el Grupo de Dartmouth recomienda introducir tópicos, conceptos y teorías en un curso preclínico y luego supervisar a los estudiantes en la aplicación del contenido y el aprendizaje apropiado de las habilidades de relación durante sus años de clínica. En 1983, consensuó los contenidos mínimos para la enseñanza de la bioética en los países de habla inglesa(2):

a) capacidad de identificar los aspectos éticos que surgen en la práctica médica; b) capacidad para obtener un consentimiento o rechazo válido de los tratamientos;

c) cómo proceder con un paciente incompetente o parcialmente competente para decidir;

d) cómo proceder si un paciente rechaza el tratamiento;

e) capacidad de decidir cuándo es moralmente justificable privar de información a un paciente;

f) capacidad de decidir cuándo es moralmente justificable romper la confidencialidad;

g) conocimiento de los aspectos éticos del cuidado de pacientes con mal pronóstico, incluyendo los enfermos terminales.

Se señalaron, además, otros dos temas que no lograron unanimidad: h) conocimiento relativo a la justa distribución de los cuidados sanitarios, e i) conocimiento referente a la interrupción voluntaria del embarazo.

Por su lado, el Grupo de Trabajo sobre la Enseñanza de la Bioética, de la Asociación de Bioética Fundamental de Madrid, propuso, en 1999, siete métodos didácticos básicos para usar en todos los niveles: clase magistral, análisis de caso, lectura crítica de texto, confección de trabajo escrito, técnicas didácticas de expresión, video foro y sesiones ético-clínicas(3).

La Conferencia Mundial de Educación Médica, de 2003, estableció los Estándares Globales en Educación Médica, señalando las competencias esenciales mínimas, entre las cuales figura, en primer lugar, la relativa a la formación ética(4):

- Valores, actitudes, conductas y ética profesional.

- Bases científicas de la medicina.

- Habilidades clínicas.

- Habilidades relacionales y comunicativas.

- Salud poblacional y sistemas asistenciales.

- Gestión de la información.

- Pensamiento crítico e investigación.

Por otro lado, la enseñanza de la bioética viene siendo introducida a ritmo creciente en los países latinoamericanos. Carlos Quintana Villar describió, en 2004, los fines de dicho esfuerzo en la Pontificia Universidad Católica de Chile(5):

- Identificar los aspectos filosóficos y éticos de la medicina.

- Conocer y familiarizarse con un modelo de análisis ético de casos clínicos.

- Conocer los comités de ética. 
- Proponer una mejor calidad de atención en salud.

- Conocer los aspectos éticos de la investigación.

- Conocer y analizar los problemas ético-clínicos del comienzo y término de la vida humana.

En Perú, el Instituto de Ética en Salud de la Facultad de Medicina de la Universidad Nacional Mayor de San Marcos, fundado en 2002, emitió opinión institucional frente al problema de la enseńanza de la ética en la Facultad de Medicina de San Fernando, recomendando lo siguiente(6):

"Enseñanza de la ética. Impartir conocimientos básicos sobre ética en el pregrado, con el fin de que el ejercicio profesional del médico se dé en un marco ético.

Respecto a la enseñanza de la ética. Mientras se adquiere la experiencia necesaria, debe hacerse en forma práctica, por lo menos dentro de los cursos fundamentales en todos los ańos de la carrera, buscando que los profesores seleccionen aquellos problemas éticos que se suscitan en cada área específica del curso que les corresponde.

La formación moral del estudiante. Se requiere de una metódica que, en realidad, aún no está disponible o no existe, señalándose la alta necesidad de investigación en este campo" (7).

De otro lado, un estudio reciente de Alarcón y colaboradores(8) analiza los currículos de 16 escuelas de medicina y seńala que un $88 \%$ de ellas dictan un solo curso de bioética, $6 \%$ dos cursos y otro $6 \%$ tres cursos. Ninguna escuela dicta el curso durante el internado. El 77\% de los cursos tienen una orientación principalmente deontológica, relegando los contenidos legales y de investigación. La metodología didáctica es a través de clases teóricas, discusión de casos y seminarios-discusión de lecturas. El contenido de tópicos de bioética en investigación representa tan sólo el 3\%. Concluyen que los contenidos de la enseńanza de bioética no se relacionan con las etapas de enseñanza de la medicina, por lo que recomiendan la incorporación de tales cursos en cada etapa, con el fin de incluir coherentemente los contenidos de investigación, deontología y aspectos legales.

Durand y colaboradores(9) estudian en los cursos de profesionales de salud de cinco escuelas los contenidos que contribuyen a la formación de valores en el estudiante, así como la percepción de éstos sobre la eficacia de estos contenidos en dicha formación. Los resultados señalan que mientras el $90 \%$ de los cursos proponen competencias y objetivos orientados a formar una escala de valores positiva y fomentar una conducta ética consecuente, los contenidos de los mismos no reflejan tal propósito. Y la percepción de los estudiantes al respecto señala un $70 \%$ de indiferencia o medianamente favorable con su dictado, expresando que, en la práctica, no se aplican tales propósitos.

\section{¿Cuáles son los objetivos de la teoría ética?}

Ortiz(10) ha señalado los siguientes:

- Como filosofía, orientada a la discusión sobre la naturaleza de los principios y las normas morales.

- Como ciencia social, orientada a la explicación de los procesos históricos de moralización de la sociedad.

- Como tecnología social, orientada a la realización efectiva de los valores máximos en la sociedad y las personas.

No obstante, para insertar todo ello en el proceso educativo que nos preocupa resulta esencial precisar qué entendemos por el concepto "hombre". Nos apoyaremos para tal finalidad en la teoría sociobiológica propuesta por $\operatorname{Ortiz}(10,11)$, quien plantea que la explicación de su naturaleza depende de cómo se resuelva la relación sociedad-conciencia-personalidad.

Esta teoría sostiene que al codificarse la información social en el neocórtex del cerebro humano éste llega a constituirse en la conciencia y, de esta manera, la actividad psíquica consciente transforma al individuo humano en una personalidad.

\section{El modelo de Ortiz acerca del desarrollo moral del hombre}

De modo muy resumido, este modelo considera que, en el sistema del universo, el sistema vivo general está constituido por sistemas vivos individuales organizados, por un lado, genéticamente a partir de su desarrollo evolutivo y, por otro, cinéticamente en virtud de los diversos tipos de información recibidos. Entre éstos, Ortiz señala la genética, la metabólica, la neural y la psíquica. En virtud de ellos, cada individuo desarrolla varios niveles de organización, de modo que la integridad de su actividad individual dependerá, fundamentalmente, de la información derivada del mayor nivel alcanzado por él en el curso de la evolución de su especie. Hasta los primates, todos los seres vivos están 
organizados por estas clases de información, y codificadas en sistemas de memoria que existen exclusivamente dentro de ellos mismos.

En el hombre, sin embargo, la situación cambia radicalmente, pues los miembros de la especie Homo Sapiens han formado, a su vez, en sentido epigenético, un sistema supra-individual. En éste se inicia un nuevo tipo de información, la información social que dará eventualmente origen al sistema que conocemos como sociedad. Cada sociedad, con su respectiva información social, habrá de reestructurar a su vez a cada individuo miembro, transformándolo gradualmente en una personalidad. Consecuentemente y en definitiva, en cada personalidad habrá de reflejarse la estructura social de la sociedad a la que pertenece y que la formó.

A esta direccionalidad de la sociedad -que incorpora en cada individuo desde el momento en que nace y en todo su desarrollo la información social que ella produce-Ortiz la denomina "determinación cinética", por contraposición a la "determinación epigenética", que depende de las bases biológicas heredadas. La información social va siendo codificada en el neocortex cerebral de cada individuo bajo la forma de información psíquica. La consecuencia de esta relación es que el hombre, a diferencia de los animales, incluso de los superiores, además de la información psíquica de tipo animal a la que puede llamarse "inconsciente", tendrá un nivel adicional de organización psíquica, de tipo personal y privado, que constituirá su información psíquica de tipo consciente y que formará su conciencia. De este modo, la información social ya almacenada como información psíquica consciente reorganizará cinéticamente todos los otros niveles de actividad del individuo: la psíquica inconsciente, la nerviosa, la metabólica y la celular, transformándolo finalmente en una personalidad.

Por otro lado, podemos diferenciar tres clases de información social que han surgido en el curso del desarrollo histórico de la sociedad: tradicional, cultural y económica, las que a su vez han determinado el desarrollo de las estructuras tradicional, cultural y económica de la sociedad, respectivamente. En virtud de ello, en cada personalidad podemos precisar tres clases de información psíquica consciente: afectiva (los sentimientos), cognitiva (los conocimientos) y conativa (las motivaciones). De éstas, para los fines del presente trabajo, nos interesan particularmente las últimas que, en síntesis, constituyen las convicciones, intereses y deseos, las intenciones, aspiraciones, expectativas y responsabilidades, los objetivos, deberes y prejuicios.

A tales convicciones las vemos estructuralmente como aquellos motivos que generan las actitudes y el carácter de la personalidad y que, procesalmente, se objetivan como valores morales desde los cuales cada ser humano, conscientemente, toma las decisiones volitivas que determinan su actuación moral o conducta. Resulta así evidente que las actitudes ante la sociedad, el trabajo, las cosas y ante sí mismo y los otros participan de una esencia moral que refleja la estructura de la sociedad en la cual el sujeto se ha desarrollado, en nuestra época con evidente influencia económica.

Los conceptos de "sociedad" y de "información social" pueden extenderse en beneficio de la tesis central del presente trabajo, pues la dinámica de la institución formadora debe reflejarse en los individuos que en ella trabajan y se forman. Por lo expuesto, también queda claro que aquello que conocemos como "conciencia moral" no es algo inmaterial o misterioso, sino el componente conativo-volitivo de la conciencia, cuya información está codificada en el sistema de memoria pre-frontal dorso-lateral del cerebro de cada persona.

Podemos imaginar la estructura de la sociedad reflejada en la estructura de la conciencia de cada hombre. Consecuentemente, si la estructura económica de una sociedad es esencialmente inmoral, ese será el modelo del componente moral de la conciencia en cada personalidad que se forme en dicha sociedad. Puede que dicho destino no tenga que darse en su totalidad en todos los individuos, pues las capacidades volitivas esencialmente morales de la personalidad de algunos pueden ser lo suficientemente fuertes y maduras como para generar, en sentido epigenético, una estructura conativo-volitiva moralmente superior a la propia sociedad. De ser así, ello se traducirá en formas de conducta igualmente superiores que, ulteriormente, pueden convertir a una persona en paradigma ético capaz de influir en la formación moral de quienes le rodean, admiran y siguen. Ejemplos de este tipo pueden hacernos recordar a Cristo, Buda, Ghandi y muchos otros.

\section{Aplicación del modelo de Ortiz a la educación humanista y ética del estudiante de medicina}

Aplicando este modelo a nuestro problema de estudio, podemos postular ahora que el estudiante de medicina, aún adolescente, se integra a una comunidad educativa 
médica universitaria, es decir, ingresa a una organización con determinada cultura y ética institucional y, posteriormente, luego de sus estudios preclínicos, lo hará a otra más compleja, el hospital. En cada una recibirá información social matizada por la cultura y ética institucional, que dependerá de todos los miembros que la componen: docentes, otros profesionales, personal administrativo y auxiliar y, finalmente, otros alumnos, adolescentes como él. Todos ellos influirán y serán influenciados por la cultura y ética institucional dominantes, las cuales, a través de la información social que generan, influirán en las respectivas conciencias morales de cada personalidad, participando como variable en el proceso de su formación o deformación profesional, según sea el caso, lo cual habrá de reflejarse en su conciencia en sus aspectos afectivo, cognitivo y conativo.

El médico desarrolla normalmente sus actividades en los medios académico y hospitalario. De prevalecer en este último una organización a la vez cohesionada y flexible, que se objetiva en una atmósfera laboral adecuada, indicativa de salud mental institucional, su adaptación no sólo será fácil sino también formativa y madurativa. Por el contrario, si la comunidad hospitalaria se halla perturbada en su desarrollo institucional, sea porque carece de normas morales precisas o porque éstas no se cumplen (hecho que suele generar conductas profesionales inadecuadas de variado nivel de severidad), al integrarse, el médico correrá el riesgo de deslizarse hacia inconductas personales. Evitar hacerlo, frente a la presión nociva de una comunidad en algún grado inmoral, creará en él un estado de tensión que, de no expresarse directamente por vías regulares, se canalizará hacia sus propios pacientes, hacia el personal de su equipo de trabajo o, peor aún, hacia su propio hogar, perturbando la relación con su propia familia.

En nuestra cultura occidental, la variable económica de poder es la que suele ejercer mayor influencia en determinar la estructura moral de la conciencia y que ésta se puede expresar en la conducta del estudiante (discente) y del médico (docente), sea por el hecho de que se hallen urgidos por lograr inmediatos ingresos para satisfacer necesidades apremiantes, sea porque en el orden económico-social actual es cada vez más difícil que quienes han elegido la profesión médica vean satisfechas sus expectativas de un nivel de vida acorde con el estatus que tradicionalmente la sociedad ha otorgado a la profesión médica.
La tesis que planteamos es que así como la sociedad determina la conciencia de la personalidad normal, así también, de estar afectada por variables de corrupción, determinará cinéticamente la estructura moral de las personalidades vulnerables, las que tendrán mayor probabilidad de adoptar conductas inmorales. El resultado siempre dependerá del balance que se establezca entre las condiciones corruptogénicas de la institución y las capacidades morales de defensa consciente del individuo, esto es, de la solidez de la escala de valores del propio discente o del propio docente. Consecuentemente, cada universidad tendrá un nivel de deformación corruptora de sus estudiantes de acuerdo a cómo esté funcionando su estructura de salud mental y de ética institucionales.

\section{Necesidad de investigación en ética y moralización del sistema formador institucional}

Ortiz critica que los esquemas propuestos para la formación del estudiante de medicina persistan en el error de un planteamiento exclusivo desde la concepción científico-natural de la especie humana o del hombreanimal. Y señala que ello torna imposible definir la educación como la ciencia del desarrollo formativo de la personalidad, impidiendo toda posibilidad de diferenciar la actuación moral de un maestro de la acción técnica de quien somete a un animal a condicionamiento experimental en un laboratorio. Por ello la calidad profesional de los egresados no ha mejorado.

En esta línea de pensamiento, y siguiendo a Ortiz, anotamos que las fallas esenciales de la educación médica en la sociedad actual no radican, contradictoriamente, en la estructura curricular, en los procedimientos usados en la enseñanza por parte de los profesores ni en las estrategias de aprendizaje empleadas por los estudiantes. El alumno de todos modos aprenderá la teoría y las destrezas médicas tanto como pueda. Recordemos la figura de los profesores de medicina de antańo quienes, aunque en desventaja por las limitaciones científicas y tecnológicas de su época, supieron formar profesionales morales con un despliegue de valores expresados en una actitud paradigmática y rectora.

La merma en la calidad moral de los nuevos profesionales de medicina obedece a una compleja trama de variables, una de las cuales corresponde a los sistemas de formación, incluyendo la selección de postulantes. 
Y al decir sistemas no sólo consideramos los planes de estudio sino el marco global del problema, desde la estructura física de la institución -con ambientes que no siempre facilitan la comunicación humana solidaria-, el apoyo de equipos adecuados para un aprendizaje teórico-práctico de calidad, la presencia de profesores informados y comprometidos con vocación de interacción humana con sus discentes, además de una estructura lógico-administrativa con autoridades competentes y honestas. Una institución de esta clase, con ética institucional bien asentada, creará un entorno en el cual la información social que discurra e influencie a sus miembros será formadora y madurativa.

Si lo mismo pudiéramos decir de los hospitales que funcionan como sedes docentes, el alumno de medicina no sólo sería reforzado en sus conocimientos científicos, sino también en sus principios y valores éticos, permitiendo una atención médica de calidad y el respeto a los derechos de los pacientes.

La observación directa nos permite señalar que éste no es el caso en muchas universidades peruanas. Por ello, no extrańa el incremento de problemas morales en los servicios médicos, que son materia de crítica y escándalo por parte de la prensa del país. El problema se agrava por la falta de una concepción integral de la naturaleza del hombre y la falta de investigación en el área, que no permiten desarrollar estrategias de corrección. Ortiz(3) señala que, desde mucho antes de enfrentar al paciente, el estudiante está convencido de que el hombre es un animal del orden de los primates, cuyo organismo debe conocer a plenitud, relegando a un plano secundario su psiquismo, no aprehendido por el método científico natural. Comparte además la falacia de que el hombre tiene una estructura psíquica emotiva y cognitiva, como todos los mamíferos. Cree también que la sociedad donde él y los demás hombres se han formado es un ambiente que en poco o nada se diferencia del ambiente de los animales. No se le enseńa, y por ende no sabe, qué es la conciencia y asume que los animales, así como los hombres, son igualmente conscientes de su entorno. Acorde con estos conceptos, su visión de la atención del paciente queda enmarcada en la idea de que la medicina es un arte y una ciencia natural, así como en la idea implícita de que la clínica no tiene tanto valor en comparación con los procedimientos instrumentales y de laboratorio.

\section{El proyecto del Instituto de Ética en Salud de la Facultad de Medicina de la Universidad Nacional Mayor de San Marcos}

Esta facultad está constituida por cinco escuelas académico-profesionales: Medicina, Enfermería, Obstetricia, Nutrición y Tecnología Médica. En 2003, Perales, Ortiz y Nolberto, utilizando metodología cualitativa y cuantitativa, estudiaron el nivel de conocimientos teóricos y experiencia docente en la enseñanza de la ética entre los profesores de la Escuela de Medicina, así como sus actitudes frente al manejo de problemas éticos en la práctica profesional. Identificaron, además, los problemas éticos más comunes en la relación docente-discente. En una primera fase y de acuerdo con cinco criterios, seleccionaron 26 profesores y dos autoridades para entrevistas en profundidad. Con los resultados, construyeron y validaron un cuestionario específico que fue aplicado luego a una muestra probabilística sistemática (29\% del total de 801 profesores). Los resultados cualitativos ubicaron diversos problemas éticos en la relación docente-alumno, entre los mismos profesores y en la práctica profesional hospitalaria, los cuales, en el criterio de los investigadores, generan modelos inadecuados de atención profesional y afectan la formación moral del estudiante.

Los resultados cuantitativos señalan que el 59\% de los profesores no ha llevado ningún curso formal de ética, deontología o bioética; sólo el 6,3\% ha llevado cursos específicos de posgrado; $84 \%$ no conoce adecuadamente cuáles son los principios de la bioética; 53\% ignora las funciones de un Comité Hospitalario de Ética; 58\% las de un Comité de Ética de Investigación y $71 \%$ el Código de Ética del Colegio Médico de Perú(12).

En 2004, Perales y Ortiz realizaron un estudio en dos hospitales públicos que funcionan como sedes docentes de varias facultades de medicina peruanas. En seminarios-talleres realizados con el personal docente de una de dichas universidades identificaron problemas éticos que agruparon en seis categorías(13):

- En la relación médico-paciente y violación de los derechos del paciente.

- En la docencia (entre otros, no se pide consentimiento informado al paciente para ser examinado por un grupo de alumnos, se discute el caso clínico en presencia del paciente).

- Relacionados con mala praxis. 
- Vinculados con las malas relaciones entre los mismos profesionales.

- En relación con actos de corrupción.

- Casos específicos de violación de principios y valores.

Sobre la base de los resultados de este estudio, Perales y colaboradores diseñaron un curso sobre Ética, Deontología y Bioética Clínica que se viene dictando a los profesores de la Escuela de Medicina desde entonces.

\section{Moralización del sistema formador institucional}

Si bien en algunas publicaciones dedicadas a la educación médica, y la de Venturelli(14) es un buen ejemplo, se insiste en la necesidad de introducir las normas éticas en el curso de la carrera, resulta evidente que la ausencia de una teoría ética cuyo objetivo sea la moralización de la sociedad explica el fracaso de tales métodos. Cuando se trata de la formación moral del estudiante, la enseńanza sigue siendo teórica e incapaz de cambiar actitudes.

Nuestro propósito central es moralizar el sistema global formador de médicos, aunque tal propuesta aparezca, en estos momentos, como un objetivo inalcanzable $(15)^{1}$. Con esto queremos adelantar la idea de que si la sociedad a la que pertenece tal sistema formador (facultad de medicina) es esencialmente injusta, está plagada de variables de corrupción y gira en torno a intereses fundamentalmente económicos no debe sorprender que las mismas variables afecten al sistema formador que en ella se inserta.

Consecuentemente, y siguiendo a Ortiz en sus postulados teóricos: "Al no disponer de una teoría del hombre, al no haber una teoría de la personalidad que explique la relación esencial que debe haber entre la estructura moral de la sociedad y la estructura motivacional del individuo, la enseñanza de la ética (sin diferenciarla de la moral) se realiza como un curso de segunda categoría: de este modo, la moral queda aislada de la vida misma, tanto del estudio del alumno, como del trabajo del profesional (...) Tenemos la convicción de que cualquier intento por introducir una estrategia educativa centrada en la formación de la persona tiene que sustentarse, aunque parezca paradójico, en una ética centrada en la sociedad (...) Creemos pues que el objetivo de la educación, en todos sus niveles y en todos los campos, debe ser la de contribuir al desarrollo formativo de cada personalidad, a pesar de las limitaciones morales de la sociedad descritas, aunque con pleno conocimiento de las razones de su existencia y poder. Pero esta explicación de la inmoralidad existente implica una estrategia educativa que persiga este objetivo en el nivel profesional, que empiece por definir y explicar de qué naturaleza es la sociedad y el sujeto que se forma dentro de ella" (16).

Recordemos las sabias palabras de Sócrates cuando sentenciaba que: "El propósito de la educación es la formación del carácter y el fin de la filosofía es la educación moral del hombre"; y las de Herbert Spencer (1820-1903): "El objetivo de la educación es la formación del carácter".

En otras palabras, desde un punto de vista ético, la institución formadora del médico debiera tener como objetivo superior no sólo capacitarlo cognitivamente en los aspectos científicos de la medicina, adiestrarlo en el desarrollo de habilidades y destrezas, tanto médicas como tecnológicas, aplicadas a la medicina, sino, especialmente, ayudarlo a formar sus convicciones y motivaciones éticas que requiere su desarrollo moral como personalidad.

Las facultades de medicina deben ser consecuentes con su responsabilidad social, produciendo el tipo de profesional que la sociedad necesita. Ello exige que sus graduados no sólo posean las características esenciales de la profesión médica, sino las específicas para su entorno cultural. Tal como atinadamente señala Pardell(17): "la profesión médica debe reconocer que su tradicional autonomía, tal como se formulara hace más de dos siglos, ya no es actualmente viable y debe aceptar una nueva formulación de sus compromisos con la sociedad en general y con el Estado en particular para que sea más explícita”.

1 Un esfuerzo valioso en la misma línea, aunque con otra metodología, es el desarrollado por el profesor Miguel Suazo, como FORO INTEC, en el Instituto Tecnológico de Santo Domingo, República Dominicana. 


\section{Referencias}

1. Equipo Fundación Educación Médica (FEM). La formación de los profesionales médicos. Monografías Humanitas 2004; 7: 69-83.

2. Culver ChM, Clouser KD, Gert B, et al. Special Report. Basic curricular goals in medical ethics. The New England Journal of Medicine 1985; 312(4): 253-256.

3. Gracia D. Enseñanza posgraduada y formación continuada en ética. Organización Médica Colegial 1992; junio-julio (21): 25-28.

4. World Federation For Medical Education. Estándares Globales en Educación Médica. Educación Médica 2004; 7 (Supl 2).

5. Quintana Villlar C. Algunos aspectos de la enseñanza de la Bioética. Boletín Escuela de Medicina. Pontificia Universidad Católica de Chile 1998; 27: 24-26.

6. Instituto de Ética en Salud. Propuesta de enseñanza de la ética médica (Bioética) en la Escuela de Medicina Humana. Lima: Facultad de Medicina, UNMSM. (documento interno); 2002.

7. Ortiz P. La Formación de la Personalidad. Lima: Dimaso E.I.R.L; 1996.

8. Alarcón J, Piscoya J, Gutiérrez C, Reyes M. Enseñanza de Bioética en las Escuelas de Medicina del Perú. Anales de la Facultad de Medicina 2005; 66(1): S58-59.

9. Durand J, Espinoza T, Del Carmen C, et al. Contenidos educativos que contribuyen en la formación de valores de los estudiantes de la Facultad de Medicina, UNMSM. Anales de la Facultad de Medicina 2005; 66(1): S58.

10. Ortiz P. Introducción a una Psicobiología del Hombre. Cuadernos de Psicobiología Social 1, Lima: Centro de Producción Editorial e Imprenta de la Universidad Nacional Mayor de San Marcos; 2004.

11. Ortiz P. Etica social, para el desarrollo moral de las instituciones educacionales y de salud. Lima: Universidad Nacional Mayor de San Marcos: 2007.

12. Perales A, Ortiz P, Nolberto V. Informe Técnico. Proyecto No 020112205. Consejo Superior de Investigaciones. Lima: Universidad Nacional Mayor de San Marcos; 2002.

13. Perales A, Ortiz P, Nolberto V. Informe Técnico. Proyecto No 040112061. Consejo Superior de Investigaciones. Lima: Universidad Nacional Mayor de San Marcos; 2004.

14. Venturelli J. Educación Médica: Nuevos enfoques, metas y métodos. Washington: Organización Panamericana de la Salud; 1997.

15. Suazo M. Bioética para nuevos. Santo Domingo: Instituto Tecnológico de Santo Domingo; 2002.

16. Ortiz P. Necesidad de un Enfoque Ético de la Educación en Medicina. Anales de la Facultad de Medicina 2001; 62: 72-81.

17. Pardell H. El nuevo profesionalismo médico: una ideología expresada en conductas. Monografías Humanitas 2004; 7: 11-22.

Recibido: 3 de diciembre de 2007

Aceptado: 18 de diciembre de 2007 